\title{
Invariant Subspaces with The Codimension One Property in $L^{t}(\mu)$
}

\author{
James E. Thomson $\&$ Liming Yang
}

Abstract. In this paper, we refine a crucial lemma in [6] (Lemma 6) and give a simple proof of it. Using this lemma, we prove that if $P^{\infty}(\mu)=H^{\infty}(D)$ and $D \backslash \operatorname{spt} \mu$ has a component whose strong boundary has positive Lebesgue measure, then each invariant subspace of $M$ of $L^{t}(\mu)$ satisfies $\operatorname{dim}(M / z M) \leq 1$. The theorem is, in some senses, a generalization of Beurling's invariant subspace theorem for Hardy spaces.

Let $H_{E}^{t}$ be the set of analytic functions on the open unit disk such that

$$
\liminf _{r \rightarrow 1-0} \int_{E}\left|f\left(r e^{i \vartheta}\right)\right|^{t} d \vartheta<\infty
$$

where $E$ is a closed subset of the unit circle with positive Lebesgue measure. Let $\mathcal{B}$ be a subset of $L_{a}^{t}(D)$ so that for each $f \in \mathcal{B}$ there exists a bounded analytic function $\varphi$ satisfying $\varphi f \in H_{E}^{t}$. Using the theorem above, we show that if $M$ is an invariant subspace of $L_{a}^{t}(D)$ generated by $\mathcal{B}$ and $E$ satisfies the Carleson condition, then $\operatorname{dim}(M / z M)=1$. Finally, we prove that there exists an irreducible subnormal operator satisfying

(1) $\sigma(S)=\{z:|z| \leq 2\}$;

(2) $\sigma_{e}(S)=\{z:|z|=1$ or $|z|=2\}$; and

(3) $\operatorname{Ind}(S-\lambda)=-1$ for $1<|\lambda|<2$ or $|\lambda|<1$.

1. Introduction. Let $\mu$ be a finite positive Borel measure with compact support in $\mathbb{C}$. For $1 \leq t<\infty$, let $P^{t}(\mu)$ denote the closure in $L^{t}(\mu)$ of polynomials in $z$. Let $P^{\infty}(\mu)$ denote the weak-star closure of polynomials in $L^{\infty}(\mu)$. If $\mu=$ $d A / \pi$, the normalized area measure of the open unit disk $D$, the space $P^{t}(d A / \pi)$ 
is called the Bergman space and denoted by $L_{a}^{t}(D)$. If $\mu=m=d \vartheta / 2 \pi$, the normalized Lebesgue measure on the unit circle, the space $P^{t}(m)$ is called the classical Hardy space and denoted by $H^{t}(D)$ (or $H^{t}$ ).

A closed subspace $M$ of $L^{t}(\mu)$ (or $P^{t}(\mu)$ ) is called an invariant subspace if $z M \subset M$. The space $M$ has the codimension- $n$ property if $\operatorname{dim}(M / z M)=n$. The structure of the lattices of invariant subspaces in Banach spaces of analytic functions has attracted a lot of attention from both operator theorists and function theorists. However, most results have been disappointing, in the sense that for most spaces there are no simple characterizations such as are known for the Hardy space $H^{t}(D)$. The famous theorem of Beurling on invariant subspaces of $H^{t}(D)$ asserts that every $z$-invariant subspace $M$ of $H^{t}(D)$ is either trivial, that is, $M=0$, or there exists an inner function $u$ such that $M=u H^{t}(D)$. In contrast to the Hardy space situation, it has been discovered by C. Apostol, H. Bercovici, C. Foias, and C. Pearcy [2] that for each $n, 1 \leq n \leq \infty$, there exist in the Bergman space invariant subspaces having the codimension- $n$ property. H. Hedenmalm [5] gave a concrete example for any $n<\infty$ of an invariant subspace having the codimension- $n$ property. Olin and Thomson $[7$, p. 425] gave an example showing that there exists an invariant subspace $M$ of $L^{2}(\mu)$, where the support of $\mu$ is contained in $\partial D \cup[0,1]$, such that $M$ has the codimension-1 property but is not generated by a single function. Aleman, Richter and Sundberg [1] show that every invariant subspace of the Bergman space $L_{a}^{2}(D)$ is generated by its wandering space. Those examples indicate that the structure of invariant subspaces should be studied in forms more general than Beurling's theorem for $H^{t}$. In this paper, we study the codimension-1 property of invariant subspaces for some function spaces. One of our theorems generalizes a result of Olin and Thomson [6, Theorem 1] from $L^{2}(\mu)$ to $L^{t}(\mu)$. Before stating our results, we need to introduce some notation.

For a finite positive Borel measure $\mu$ with compact support in the complex plane, let spt $\mu$ denote the support of $\mu$. The Cauchy transform of $\mu$, denoted by $\hat{\mu}$, is defined by

$$
\hat{\mu}(\lambda)=\int \frac{1}{z-\lambda} d \mu .
$$

The function is locally integrable with respect to area measure.

Let $W$ be a connected component of $D \backslash \operatorname{spt} \mu$. Olin and Thomson [6] have defined the strong boundary of $W, B_{W}$, to be the set of points $a$ in $\partial D \cap \partial W$ so that for all $\alpha \in(0, \pi)$, there is an isosceles triangle $T_{a, \alpha}^{s}$ so that

(1) $a$ is a vertex of $T_{a, \alpha}^{s}$ and $s$ is the height of $T_{a, \alpha}^{s}$;

(2) $\operatorname{int} T_{a, \alpha}^{s} \subset W$;

(3) The interior angle of $T_{a, \alpha}^{s}$ at $a$ is $\alpha$;

(4) The radial line segment from 0 to $a$ bisects the interior angle of $T_{a, \alpha}^{s}$ at $a$. 
The strong boundary of $W$ is a Borel set (see [6]). If $m\left(B_{W}\right)>0$, we call $W$ an outer hole of the support of $\mu$.

Let $A(D)$ be the disk algebra, that is, the algebra of analytic functions in $D$ that are continuous on the closed unit disk. Let $E$ be a compact subset of the unit circle and $\partial D \backslash E=\bigcup J_{n}$, where the open $\operatorname{arc} J_{n}$ is a connected component. Say $E$ satisfies the Carleson condition if

$$
\sum m\left(J_{n}\right) \log \frac{1}{m\left(J_{n}\right)}<\infty
$$

Let $H_{E}^{t}$ be the space of analytic functions $f$ in $D$ such that

$$
\liminf _{r \rightarrow 1-} \int_{E}\left|f\left(r e^{i \vartheta}\right)\right|^{t} d \vartheta<\infty
$$

Let $\mathcal{H}$ be a separable Hilbert space on $\mathbb{C}$. A bounded linear operator $S$ on $\mathcal{H}$ is called a subnormal operator if there exists a normal operator $N$ on a Hilbert space $\mathcal{K}$ containing $\mathcal{H}$ such that $N \mathcal{H} \subset \mathcal{H}$ and $S=\left.N\right|_{\mathcal{H}}$. The operator $S$ is irreducible if $S$ has no non-trivial reducing subspaces.

In Section 2, we refine a crucial lemma (see Lemma 2.1) in [6] and give a simple proof of it (see [6, pp. 122-132] for the original proof). Using this result, we are able to generalize Theorem 1 of [6] to the following theorem.

Theorem A. Suppose that $P^{\infty}(\mu)=H^{\infty}(D)$. For $1<t<\infty$ let $M$ be an invariant subspace of multiplication by $z$ on $L^{t}(\mu)$ and let $U$ be an outer hole of $\operatorname{spt} \mu$ with $0 \in U$. Then $\operatorname{dim}(M / z M) \leq 1$.

In Section 3, using Theorem A, we prove the following theorem:

Theorem B. Let a compact subset $E \subset \partial D$ of positive Lebesgue measure satisfy the Carleson condition, and let $\mathcal{B}$ be a subset of $L^{t}(D)(1<t<\infty)$. Suppose that for each $f \in \mathcal{B}$ there exists a bounded analytic function $\varphi$ such that $\varphi f \in H_{E}^{t}$. Let $M$ be the invariant subspace generated by $\mathcal{B}$. Then $M$ has the codimension-1 property.

The case where $p=2$ was done in [9].

Finally, in Section 4, we prove the following theorem, which answers a question of J. B. Conway.

Theorem C. There exists an irreducible subnormal operator $S$ such that

(1) $\sigma(S)=\{z:|z| \leq 2\}$;

(2) $\sigma_{e}(S)=\{z:|z|=1\} \cup\{z:|z|=2\}$; and

(3) $\operatorname{Ind}(S-\lambda)=-1$ for each $\lambda \in\{z: 1<|z|<2\} \cup\{z:|z|<1\}$. 
2. Invariant subspaces of $L^{t}(\mu)$. The following lemma estimates the Cauchy transform of a measure inside an outer hole. The lemma is slightly different from a crucial lemma (Lemma 6) in [6]; however, we will provide a simple and direct proof. We will use letters $c$ and $C$ to denote constants that may change from one step to the next.

Lemma 2.1. Suppose that $P^{\infty}(\mu)=H^{\infty}(D), g \in L^{1}(\mu), z g d \mu \perp A(D)$ and $|g| d \mu$ is a Carleson measure. Let $U$ be an outer hole of $\operatorname{spt} \mu$ with $m\left(B_{U}\right)>$ 0 . Then there exists a region $V \subset U$ with rectifiable boundary $\partial V, m\left(B_{V}\right)>0$, and $\widehat{z g d \mu}$ is a bounded analytic function on $V$. Moreover, for some $\beta>0$,

$$
\lim _{r e^{i \vartheta} \in \Lambda_{\vartheta_{0}, \beta} \rightarrow e^{i \vartheta_{0}}} \widehat{z g d \mu}\left(r e^{i \vartheta}\right)=g\left(e^{i \vartheta_{0}}\right) \frac{d \mu}{d m}\left(e^{i \vartheta_{0}}\right)
$$

a.e. with respect to $m$ on $B_{V}$, where $\Lambda_{\vartheta, \beta}$ denote the Stoltz angle at $e^{i \vartheta}$ with opening $2 \beta$.

Proof. Choose a compact subset $K$ of $B_{U}$ with $m(K)>0$ and $N<\infty$ such that

$$
\left|g\left(e^{i \vartheta}\right)\right| \frac{d \mu}{d m}\left(e^{i \vartheta}\right) \leq N
$$

on $K$.

Set

$$
\Lambda_{\vartheta, \alpha}^{r}=\Lambda_{\vartheta, \alpha} \cap\{z:|z|>r\} .
$$

Fix $\pi / 3 \leq \alpha<\pi / 2$. For each $e^{i \vartheta} \in K$ there exists $r_{\vartheta}$ such that $\Lambda_{\vartheta, \alpha}^{r_{\vartheta}} \subset U$. Set

$$
G=\bigcup_{e^{i \vartheta} \in K} \Lambda_{\vartheta, \alpha}^{r_{\vartheta}}=\bigcup G_{n}
$$

where $G_{n}$ is a connected component of $G$. It is obvious that

$$
K \subset \bigcup\left(\partial G_{n} \cap \partial D\right)
$$

Hence, there exists $G_{n}$, call it $W$, such that $m(\partial W \cap \partial D)>0$ and $\partial W$ is a rectifiable Jordan curve. So $m\left(B_{W}\right)>0$ (see [4, p. 45]). Let $\partial D \backslash \partial W=\bigcup I_{n}$, where $I_{n}$ denotes an open arc. Let $L W$ denote the set of $e^{i \vartheta} \in B_{W}$ such that

$$
\lim _{\delta \rightarrow 0} \frac{m\left(\partial D \cap B_{W} \cap(\vartheta-\delta, \vartheta+\delta)\right)}{2 \delta}=1 .
$$

Let $\left[\vartheta_{1}, \vartheta_{2}\right]$ be the smallest closed arc containing $\partial W \cap \partial D$. 
Claim. There exists $C>0$ with the following properties: if $e^{i \vartheta_{0}} \in L W$ and $\lambda=r e^{i \vartheta} \in \Lambda_{\vartheta_{0}, \beta}(\beta<\alpha)$, then there is a positive number $\delta_{\vartheta_{0}}<$ $\min \left(\left|\vartheta_{1}-\vartheta_{0}\right|,\left|\vartheta_{2}-\vartheta_{0}\right|\right)$ such that

$$
\int_{D_{\vartheta_{0}} \cap D}\left|\frac{z}{z-\lambda}-\frac{z}{z-1 / \bar{\lambda}}\right||g| d \mu \leq C \int_{\cup I_{n}} P_{r}(t-\vartheta) d t
$$

where $D_{\vartheta_{0}}=\left\{z:\left|z-e^{i \vartheta_{0}}\right| \leq \delta_{\vartheta_{0}}\right\}$ and $P_{r}(t-\vartheta)$ is the Poisson kernel at $\lambda$.

Let $I_{n}=\left(a_{n}, b_{n}\right)$ and $\Delta_{n}$ be the closed region bounded by $I_{n}, \partial \Lambda_{a_{n}, \alpha}$ and $\partial \Lambda_{b_{n}, \alpha}$. By our assumption, when $I_{n}$ is close to $e^{i \vartheta_{0}},\left|I_{n}\right|$ is much smaller than $\left|b_{n}-\vartheta_{0}\right|$. Hence we can choose $\delta_{\vartheta_{0}}>0$ so that if $\lambda \in \Lambda_{\vartheta_{0}, \beta}(\beta<\alpha)$ and $z \in \Delta_{n}$, where $\Delta_{n} \cap D_{\vartheta_{0}} \neq \varnothing$, then $\left|I_{n}\right| \leq \frac{1}{2}|z-\lambda|$, and so

$$
\frac{1}{2} \leq \frac{\left|e^{i t}-\lambda\right|-\left|I_{n}\right|}{\left|e^{i t}-\lambda\right|} \leq \frac{|z-\lambda|}{\left|e^{i t}-\lambda\right|} \leq \frac{\left|e^{i t}-\lambda\right|+\left|I_{n}\right|}{\left|e^{i t}-\lambda\right|} \leq 2
$$

and similarly,

$$
\frac{1}{2} \leq \frac{\left|e^{i t}-1 / \bar{\lambda}\right|-\left|I_{n}\right|}{\left|e^{i t}-1 / \bar{\lambda}\right|} \leq \frac{|z-1 / \bar{\lambda}|}{\left|e^{i t}-1 / \bar{\lambda}\right|} \leq \frac{\left|e^{i t}-1 / \bar{\lambda}\right|+\left|I_{n}\right|}{\left|e^{i t}-1 / \bar{\lambda}\right|} \leq 2
$$

for $t \in I_{n}$. Thus, for $t \in I_{n}, z \in \Delta_{n}\left(\Delta_{n} \cap D_{\vartheta_{0}} \neq \varnothing\right)$ and $\lambda \in \Lambda_{\vartheta_{0}, \beta}$,

$$
\begin{aligned}
\left|\frac{z}{z-\lambda}-\frac{z}{z-1 / \bar{\lambda}}\right| & \leq C\left|\frac{e^{i t}}{e^{i t}-\lambda}-\frac{e^{i t}}{e^{i t}-1 / \bar{\lambda}}\right| \\
& =C P_{r}(t-\vartheta) .
\end{aligned}
$$

Therefore,

$$
\begin{aligned}
\int_{\Delta_{n}}\left|\frac{z}{z-\lambda}-\frac{z}{z-1 / \bar{\lambda}}\right| g \mid d \mu & \leq C \inf _{t \in I_{n}} P_{r}(t-\vartheta) \int_{\Delta_{n}}|g| d \mu \\
& \leq C \inf _{t \in I_{n}} P_{r}(t-\vartheta)\left|I_{n}\right| \\
& \leq C \int_{I_{n}} P_{r}(t-\vartheta) d t
\end{aligned}
$$

since $|g| d \mu$ is a Carleson measure and $\Delta_{n}$ is contained in a Carleson square with length $\left|I_{n}\right|$. Now the claim follows since

$$
D \cap \operatorname{spt} \mu \cap D_{\vartheta_{0}} \subset \bigcup\left(\Delta_{n} \cap D_{\vartheta_{0}}\right)
$$


Now take a point $e^{i \vartheta_{0}} \in L W$ so that

$$
\lim _{r e^{i \vartheta} \in \Lambda_{\vartheta_{0}, \beta} \rightarrow e^{i \vartheta_{0}}} \int_{\partial D} P_{r}(t-\vartheta) g\left(e^{i t}\right) d \mu(t)=g\left(e^{i \vartheta_{0}}\right) \frac{d \mu}{d m}\left(e^{i \vartheta_{0}}\right) .
$$

From the claim and Fatou's Theorem (see [3, p. 111]),

$$
\begin{aligned}
& \lim _{r e^{i \vartheta} \in \Lambda_{\vartheta_{0}, \beta} \rightarrow e^{i \vartheta_{0}}} \int_{D_{\vartheta_{0}} \cap D}\left|\frac{z}{z-\lambda}-\frac{z}{z-1 / \bar{\lambda}}\right||g| d \mu \\
\leq & \lim _{\delta \rightarrow 0} \frac{m\left(\bigcup I_{n} \cap(\vartheta-\delta, \vartheta+\delta)\right)}{2 \delta}=0 .
\end{aligned}
$$

Therefore,

$$
\begin{aligned}
& \widehat{z g d \mu}(\lambda)= \int\left(\frac{z}{z-\lambda}-\frac{z}{z-1 / \bar{\lambda}}\right) g d \mu \\
&= \int_{D \backslash D_{\vartheta_{0}}}\left(\frac{z}{z-\lambda}-\frac{z}{z-1 / \bar{\lambda}}\right) g d \mu+\int_{D_{\vartheta_{0}} \cap D}\left(\frac{z}{z-\lambda}-\frac{z}{z-1 / \bar{\lambda}}\right) g d \mu \\
& \quad+\int_{\partial D} P_{r}(t-\vartheta) g\left(e^{i t}\right) d \mu(t) \\
& \rightarrow g\left(e^{i \vartheta_{0}}\right) \frac{d \mu}{d m}\left(e^{i \vartheta_{0}}\right)
\end{aligned}
$$

when $\lambda=r e^{i \vartheta} \in \Lambda_{\vartheta_{0}, \beta} \rightarrow e^{i \vartheta_{0}}$. Therefore, we can choose $r>0$ such that

$$
|\widehat{z g d \mu}(\lambda)| \leq N+1 \quad \text { on } \Lambda_{\vartheta_{0}, \beta}^{r} \text {. }
$$

Set $V_{0}=\bigcup_{e^{i \vartheta_{0} \in L W}} \Lambda_{\vartheta_{0}, \beta}^{r}=\bigcup V_{n}$, where $V_{n}$ is a connected component of $V_{0}$. As before, we can choose one $V_{n}$, call it $V$, so that $m\left(B_{V}\right)>0, \partial V$ is a rectifiable Jordan curve, and on $V$ we know that $\widehat{z g d \mu}$ is bounded by $N+1$.

Remark. From the proof of the lemma, one sees that the region $V$ can be chosen such that $m\left(B_{U} \backslash B_{V}\right)$ is arbitarily small.

Theorem 2.2. $\quad$ Suppose that $P^{\infty}(\mu)=H^{\infty}(D)$. For $1<t<\infty$ let $M$ be an invariant subspace of multiplication by $z$ on $L^{t}(\mu)$ and let $U$ be an outer hole of $\operatorname{spt} \mu$ with $0 \in U$. Then $\operatorname{dim}(M / z M) \leq 1$.

Proof. Suppose that $\operatorname{dim}(M / z M) \geq 2$. Then there exists two linearly independent functionals $L_{1}, L_{2} \in M^{*}$ such that $\left\|L_{i}\right\|=1$ and $L_{i}(z x)=0$, for $x \in M$ and $i=1,2$. Using the Hahn-Banach theorem, we extend $L_{1}$ and $L_{2}$ to two unit functionals on $L^{t}(\mu)$. By Riesz's representation theorem there exist two 
functions $g_{1}$ and $g_{2}$ in $L^{t^{\prime}}(\mu)$, where $1 / t+1 / t^{\prime}=1$, such that $\left\|g_{1}\right\|_{t^{\prime}}=\left\|g_{2}\right\|_{t^{\prime}}=1$ and

$$
L_{i}(x)=\int x g_{i} d \mu, \quad \text { for } x \in M \text { and } i=1,2 .
$$

Since

$$
1=\left\|L_{i}\right\|=\sup _{\|x\|_{t}=1, x \in M}\left|\int x g_{i} d \mu\right|,
$$

we can find $x_{i} \in M$ such that $\left\|x_{i}\right\|_{t}=1$ and

$$
\int x_{i} g_{i} d \mu=1
$$

(The existence of such an $x_{i}$ is guaranteed by the assumption that $1<p<\infty$, and the weak compactness of the unit ball of $\left.L^{p}(\mu)\right)$. It follows from Hölder's inequality that

$$
1=\int x_{i} g_{i} d \mu \leq\left\|x_{i}\right\|_{t}\left\|g_{i}\right\|_{t^{\prime}}=1
$$

Hence, Hölder inequality becomes an equality in our case. Therefore,

$$
x_{i} g_{i}=\left|x_{i}\right|^{t}=\left|g_{i}\right|^{t^{\prime}} \quad \text { a.e. } \mu .
$$

Claim. $\operatorname{det}\left[\int x_{i} g_{j} d \mu\right] \neq 0$.

Suppose not. There exist two constants $a$ and $b$ (one of them is nonzero) such that

$$
a \int x_{1} g_{1} d \mu+b \int x_{1} g_{2} d \mu=0
$$

and

$$
a \int x_{2} g_{1} d \mu+b \int x_{2} g_{2} d \mu=0 .
$$

It follows from Hölder's inequality that

$$
|a|=|b|\left|\int x_{1} g_{2} d \mu\right| \leq|b|\left\|x_{1}\right\|_{t}\left\|g_{2}\right\|_{t^{\prime}}=|b|
$$

and

$$
|b|=|a|\left|\int x_{2} g_{1} d \mu\right| \leq|a|\left\|x_{2}\right\|_{t}\left\|g_{1}\right\|_{t^{\prime}}=|a| .
$$

Hence,

$$
\left|\int x_{1} g_{2} d \mu\right|=\left\|x_{1}\right\|_{t}\left\|g_{2}\right\|_{t^{\prime}}
$$


Therefore, there exists a constant $c$ with $|c|=1$ such that

$$
c x_{1} g_{2}=\left|x_{1}\right|^{t}=\left|g_{2}\right|^{t^{\prime}} \quad \text { a.e. } \mu \text {. }
$$

It follows from (1) that $g_{1}=c g_{2}$. This is a contradiction. The claim is established.

Let $G_{i j}(\lambda)=z \widehat{x_{i} g_{j}} d \mu(\lambda)$. It follows from our construction that

$$
z\left|x_{i}\right|^{t} d \mu \perp A(D)
$$

and $\left|x_{i}\right|^{t} d \mu \perp A_{0}(D)$. Therefore, $\left|x_{i}\right|^{t} d \mu$ is a Carleson measure (same proof of Lemma 2 in [6]). From our construction and Hölder's inequality, we see that $z x_{i} g_{j} d \mu \perp A(D)$ and $\left|x_{i} g_{j}\right| d \mu$ is a Carleson measure. Hence, it follows from Lemma 2.1 that $G_{i j}(\lambda)$ is a bounded analytic function on $V$ ( $V$ comes from Lemma 2.1, we may assume that $0 \in V$ ) and

$$
\lim _{r \rightarrow 1-0} G_{i j}\left(r e^{i \vartheta}\right)=x_{i}\left(e^{i \vartheta}\right) g_{j}\left(e^{i \vartheta}\right) \frac{d \mu}{d m}\left(e^{i \vartheta}\right)
$$

a.e. on $B_{V}$. Thus,

$$
\lim _{r \rightarrow 1-0} G_{11} G_{22}\left(r e^{i \vartheta}\right)=\lim _{r \rightarrow 1-0} G_{21} G_{12}\left(r e^{i \vartheta}\right)
$$

a.e. on $B_{V}$. Therefore, $G_{11}(\lambda) G_{22}(\lambda)=G_{12}(\lambda) G_{12}(\lambda)$ on $V$ since they are bounded analytic functions. This contradicts our claim if we take $\lambda$ to be zero.

A point $\lambda \in \mathbb{C}$ is a bounded point evaluation (bpe) for $P^{t}(\mu)$ if there exists $C>0$ such that

$$
|p(\lambda)| \leq C\|p\|_{t} .
$$

Corollary 2.3. Let $1<t<\infty$ and let $\mu$ be a finite positive Borel measure with support in the closed unit disk. Suppose that the set of bpe's for $P^{t}(\mu)$ is the open unit disk and $\operatorname{spt} \mu$ has an outer hole. Then every invariant subspace of $P^{t}(\mu)$ has the codimension-1 property.

3. Invariant subspaces of the Bergman space. In [9], Theorem B was proved in the case where $t=2$. The main difficulty there was to construct an outer hole for a certain measure. This turns out to be true for $1 \leq t<\infty$. We will not give the proof for constructing such an outer hole, however, we will state the facts that we need in the following two lemmas (see Section 2 of [9] for details). 
Lemma 3.1. Let $1 \leq t<\infty$. Let $E$ be a closed subset of the unit circle with $m(E)>0$. Let $\mu_{E}$ be the area measure inside the open unit disk and Lebesgue measure on E. Suppose that E satisfies the Carleson condition. There exists an outer hole $W$ of the measure $\mu_{E}^{W}=\left.\mu_{E}\right|_{\bar{D} \backslash W}$ such that

$$
c \int|p|^{t} d \mu_{E} \leq \int|p|^{t} d \mu_{E}^{W} \leq \int|p|^{t} d \mu_{E}
$$

for each polynomial $p$.

Lemma 3.2. $\quad$ Suppose that $W$ is an outer hole in Lemma 3.1. Then $L_{a}^{t}(D) \cap H_{E}^{t}$ is contained in $P^{t}\left(\mu_{E}^{W}\right)$.

Theorem 3.3. Let a compact subset $E \subset \partial D$ of positive Lebesgue measure satisfy the Carleson condition, and let $\mathcal{B}$ be a subset of $L^{t}(D)(1<t<\infty)$. Suppose that for each $f \in \mathcal{B}$ there exists a bounded analytic function $\varphi$ such that $\varphi f \in H_{E}^{t}$. Let $M$ be the invariant subspace generated by $\mathcal{B}$. Then $M$ has the codimension-1 property.

Proof. Let $W$ be the outer hole in Lemma 3.1 Without loss of generality, we assume $0 \in W$. Using Corollary 3.15 of [8], we assume that $M$ is generated by two functions $f_{1}$ and $f_{2}$. There exists two bounded analytic functions $\varphi_{1}$ and $\varphi_{2}$ (we may assume $\varphi_{1}(0) \neq 0 \neq \varphi_{2}(0)$ ) such that $\varphi_{i} f_{i} \in H_{E}^{t}$ for $i=1,2$. Using Lemma 3.2, we see that $\varphi_{i} f_{i} \in P^{t}\left(\mu_{E}^{W}\right)$. Let $N$ and $M_{0}$ be the invariant subspace generated by $\varphi_{1} f_{1}$ and $\varphi_{2} f_{2}$ in $P^{t}\left(\mu_{E}^{W}\right)$ and $L_{a}^{t}(D)$, respectively. From Lemma 3.1,

$$
\int|f|^{t} d A \leq C \int|f|^{t} d \mu_{E}^{W}
$$

for each $f \in N$. So we can define a bounded linear operator $X$ from $N$ to $M_{0}$ by $X f=f . X$ has dense range and maps $z N$ into $z M_{0}$. Hence, we can define an induced operator $[X]$ from $N / z N$ to $M_{0} / z M_{0}$ by $[X][f]=[f]$. It is easy to check $[X]$ is bounded with dense range. It follows from Corollary 2.3 that $\operatorname{dim}(N / z N)=1$. Hence, $\operatorname{dim}\left(M_{0} / z M_{0}\right)=1$.

Now suppose that $\operatorname{dim}(M / z M)=2$. We choose $g_{1}, g_{2} \in L_{a}^{t^{\prime}}(D)$ such that

(a) $\operatorname{det}\left[\int f_{i} g_{j} d A\right] \neq 0$;

(b) $\int z f_{i} g_{j} d A=0$ for each $f \in M$.

However,

$$
\operatorname{det}\left[\int \varphi_{i} f_{i} g_{j} d A\right]=\varphi_{1}(0) \varphi_{2}(0) \operatorname{det}\left[\int f_{i} g_{j} d A\right] \neq 0 .
$$

Therefore, $\operatorname{dim}\left(M_{0} / z M_{0}\right)=2$. This is a contradiction. 
4. An irreducible subnormal operator. In this section, we construct an irreducible subnormal operator satisfying certain conditions in the following theorem.

Theorem 4.1. There exists an irreducible subnormal operator $S$ such that

(1) $\sigma(S)=\{z:|z| \leq 2\}$;

(2) $\sigma_{e}(S)=\{z:|z|=1\} \cup\{z:|z|=2\}$; and

(3) $\operatorname{Ind}(S-\lambda)=-1$ for each $\lambda \in\{z: 1<|z|<2\} \cup\{z:|z|<1\}$.

Proof. Let $A=\{z: 1 \leq|z| \leq 2\}$, let $m_{1}$ be the Lebesgue measure on the unit circle and let $m_{2}$ be the Lebesgue measure on $\{z:|z|=2\}$. Let $K$ be a compact subset of the unit circle with $m_{1}(K)>0$ and such that $K$ has no interior. Let $R^{2}\left(A, m_{1}+m_{2}\right)$ be the closure in $L^{2}\left(m_{1}+m_{2}\right)$ of rational functions with poles off $A$ and let $H^{2}\left(m_{1}\right)$ be the usual Hardy space on the unit circle. Set

$\mathcal{H}=\left\{(f, g) \in R^{2}\left(A, m_{1}+m_{2}\right) \oplus H^{2}\left(m_{1}\right): f\left(e^{i \vartheta}\right)=g\left(e^{i \vartheta}\right)\right.$ a.e. $m_{1}$ for $e^{i \vartheta}$ in $\left.K\right\}$.

Obviously, $\mathcal{H}$ is a closed subspace of $R^{2}\left(A, m_{1}+m_{2}\right) \oplus H^{2}\left(m_{1}\right)$ and $z \mathcal{H} \subset \mathcal{H}$. Let $S$ be the multiplication by $z$ on $\mathcal{H}$. Then $S$ is an irreducible subnormal operator. In fact, if $S$ were not irreducible, there would be $\left(f_{1}, g_{1}\right),\left(f_{2}, g_{2}\right) \in \mathcal{H}$ such that

$$
\left\langle p\left(f_{1}, g_{1}\right), q\left(f_{2}, g_{2}\right)\right\rangle=0
$$

for all polynomials $p, q$. Hence, $f_{1}\left(e^{i \vartheta}\right) \bar{f}_{2}\left(e^{i \vartheta}\right)+g_{1}\left(e^{i \vartheta}\right) \bar{g}_{2}\left(e^{i \vartheta}\right)=0$ a.e. $m_{1}$ on $K$. Thus, $g_{1}$ or $g_{2}$ will vanish on a positive Lebesgue measure set of the unit circle. So one of $\left(f_{1}, g_{1}\right)$ and $\left(f_{2}, g_{2}\right)$ will be zero. This is a contradiction.

(1) is obviously true. For $(3)$, take $1<|\lambda|<2$. For each $(f, g) \in \mathcal{H}$, we have

$$
(f, g)-f(\lambda)(1,1)=(z-\lambda)(F, G)
$$

where $F=(f-f(\lambda)) /(z-\lambda)$ and $G=(g-f(\lambda)) /(z-\lambda)$ and $(F, G)$ is in $\mathcal{H}$. For $|\lambda|<1$ we have a similar argument. Hence,

$$
\sigma_{e}(S) \subset\{z:|z|=1 \text { or }|z|=2\}
$$

and $\operatorname{Ind}(S-\lambda)=-1$ for $\lambda \in\{z: 1<|z|<2$ or $|z|<1\}$.

For (2), take $\lambda=e^{i \vartheta_{0}} \in \partial D \backslash K$. Denote $I_{n}=\left\{e^{i \vartheta}:\left|\vartheta-\vartheta_{0}\right|<1 / n\right\}$. For $n$ large enough, $I_{n} \subset \partial D \backslash K$. Let $\varphi_{n}$ be the Riemann map from $S^{2} \backslash I_{n}$ to the unit disk $D$ with $\varphi_{n}(\infty)=0$. It is easy to see that $\varphi_{n}^{\prime}(\infty)$ is comparable with $1 / n$, so by the maximum modulus theorem the function $\left(z-e^{i \vartheta_{0}}\right) \varphi_{n}(z) / \varphi_{n}^{\prime}(\infty)$ is uniformly bounded and equals 1 at $\infty$. By a normal family argument, we may 
assume that $\left(z-e^{i \vartheta_{0}}\right) \varphi_{n}(z) / \varphi_{n}^{\prime}(\infty)$ converges to 1 pointwisely on $S^{2} \backslash\left\{e^{i \vartheta_{0}}\right\}$. Let $f_{n}=\varphi_{n}(z) / \varphi_{n}^{\prime}(\infty)$, then

$$
\int\left|f_{n}\right|^{2} d m_{1} \geq \int_{I_{n}}\left|f_{n}\right|^{2} d m_{1} \geq c n \rightarrow \infty
$$

and $\left(f_{n}, f_{n}\right) \in \mathcal{H}$. Moreover, the sequence $\left\{\left(z-e^{i \vartheta_{0}}\right)\left(f_{n}, f_{n}\right)\right\}$ converges to $(1,1)$. Let $g_{n}=\left(f_{n}, f_{n}\right) /\left\|\left(f_{n}, f_{n}\right)\right\|$, then it is easy to show that

$$
\lim _{n \rightarrow \infty}\left\|\left(z-e^{i \vartheta_{0}}\right) g_{n}\right\|=0
$$

Thus, $e^{i \vartheta_{0}} \in \sigma_{e}(S)$. Since $\sigma_{e}(S)$ is closed, (2) follows.

Remark. The problem in this section was pointed out to us by John B. Conway. The authors wish to express their gratitude to him.

\section{REFERENCES}

[1] A. Aleman, S. Richter \& C. Sundberg, Beurling's theorem for the Bergman space, (Preprint).

[2] C. Apostol, H. Bercovici, C. Foias \& C. PeArcy, Invariant subspaces, dilation theory, and the structure of the predual of a dual algebra, I, J. Funct. Anal. 63 (1985), 369-404.

[3] J. B. Conway, The theory of subnormal operators, Math. Surveys Monographs, Vol 36, Amer. Math. Soc., Providence, RI, 1991.

[4] P. L. Duren, Theory of $H^{p}$ spaces, Academic Press, New York, 1970.

[5] H. Hedenmalm, An invariant subspace of the Bergman space having the codimension two property, J. Reine Angew. Math 443 (1993), 1-9.

[6] R. Olin \& J. Thomson, Some index theorems for subnormal operators, J. Operator Theory 3 (1980), 115-142.

[7] Cellular-indecomposable subnormal operators, Integral Equations Operator Theory 7 (1984), 392-430.

[8] S. Richter, Invariant subspaces in Banach spaces of analytic functions, Trans. Amer. Math. Soc. 304 (1987), 585-616.

[9] L. YANG, Invariant subspaces of the Bergman space and some subnormal operators in $\mathcal{A}_{1} \backslash \mathcal{A}_{2}$, Michigan Math. J. 42 (1995) (to appear).

The second author was partially supported by NSF grant DMS-9401234.

JAMES E. THOMSON

Department of Mathematics

Virginia Polytechnic Institute and State University

Blacksburg, Virginia 24061
LIMING YANG

Department of Mathematics

University of Hawaii

Honolulu, Hawaii 96822

Received: October 6th, 1995. 